\title{
Identification of $\mathrm{CD4}^{+} \mathrm{CD}^{+} 5^{+} \mathrm{CD} 127^{-}$regulatory $\mathrm{T}$ cells and $\mathrm{CD}^{+}{ }^{+}{ }^{-H L A}{ }^{-D R} /$ low myeloid-derived suppressor cells and their roles in the prognosis of breast cancer
}

\author{
JINHU WANG and JIANHONG YANG
}

Department of Laboratory Medicine, Taicang Affiliated Hospital of Soochow University, Taicang, Jiangsu 215400, P.R. China

Received September 30, 2015; Accepted January 18, 2016

DOI: $10.3892 /$ br.2016.694

\begin{abstract}
The aim of the present study was to identify cluster of differentiation $4^{+}\left(\mathrm{CD} 4^{+}\right) \mathrm{CD} 25^{+} \mathrm{CD} 127^{-}$regulatory $\mathrm{T}$ cells (Tregs) and $\mathrm{CD} 14^{+}$human leukocyte antigen-antigen D-related (HLA-DR-)/low myeloid-derived suppressor cells (MDSCs) in patients with breast cancer of varying stages, and investigate their roles and the potential interactions in the prognosis of breast cancer. A total of 40 patients with breast cancer were included in the study. A total of 30 healthy individuals served as the healthy control. Flow cytometry was performed for the identification of biomarkers. Natural Tregs were characterized by the expression of $\mathrm{CD} 4{ }^{+} \mathrm{CD} 25^{+} \mathrm{CD} 127^{-}$. The MDSC frequency was expressed as the percentage of $\mathrm{CD}_{3}{ }^{+} \mathrm{CD}_{11 b^{+} \mathrm{HLA}^{-}{ }^{-} \mathrm{R}^{-} \text {lineage markers (Lin) }}{ }^{-}$. The absolute number of Tregs was higher in breast cancer patients compared to the healthy control. The absolute number of Tregs in the patients with stage III or IV breast cancer was higher than those of the stage I or II, respectively. The percentage showed a gradual increase in the patients with breast cancer compared with the normal control. No direct correlation was established between the number or percentage of Tregs and the patient survival. There was a higher percentage of circulating MDSCs in breast cancer patients compared with the normal individuals. A close correlation was established between clinical cancer stage and percentage and total number of circulating MDSCs. To be exact, a significant increase of MDSC percentage and total number was observed in patients with stage III-IV breast cancer compared with the other cancer patients (stage I-II) and the normal individuals. No statistical difference was observed in the 3-and 5-year survival rates in the breast cancer patients with enhanced expression of Tregs, compared with the normal individuals. In conclusion, enhanced expression of $\mathrm{CD} 4^{+} \mathrm{CD} 25^{+} \mathrm{CD} 127^{-}$Tregs cells and
\end{abstract}

Correspondence to: Dr Jinhu Wang, Department of Laboratory Medicine, Taicang Affiliated Hospital of Soochow University, 58 Changshengnan Road, Taicang, Jiangsu 215400, P.R. China E-mail: jstcyyjyk1@sina.com

Key words: breast cancer, T-regulatory cells, myeloid-derived suppressor cells, prognosis
CD $33{ }^{+} \mathrm{CD} 11^{+} \mathrm{HLA}^{-} \mathrm{DR}-\mathrm{LIN}^{-}$MDSCs were identified from patients with breast cancer. Patients with advanced stage breast cancer showed upregulation of such cells. However, these 2 types of cells showed no correlation with the prognosis of breast cancer.

\section{Introduction}

Breast cancer is a malignant carcinoma commonly reported in women, causing a large threat to public health worldwide (1). According to the International Agency for Research on Cancer, a total of 1.4 million women are suffering from breast cancer annually, with a mortality of up to $33 \%$ (2). In China, breast cancer has been considered as the second most lethal disease for women following pulmonary carcinoma.

The pathogenesis of tumors has been considered as associated with different mechanisms to evade the immune response of the host, thus generating a suppressive network (3). Currently, immunotherapy has been well acknowledged as a promising treatment modality for numerous types of cancer (4). Regulatory T cells (Tregs), also known as cluster of differentiation $4^{+}\left(\mathrm{CD} 4^{+}\right) \mathrm{CD} 25^{+}$Tregs, are a population of cells produced by the normal thymus and have key roles in the maintenance of immune responses. Natural Tregs are highly dependent on the expression of Foxp3, which is crucial for controlling their development and function in a highly Treg-specific manner (5). Foxp3 is known as a reliable marker of Tregs. Therefore, it is possible to define Tregs more strictly as $\mathrm{CD} 4^{+} \mathrm{CD} 25^{+} \mathrm{Foxp} 3^{+}$ cells (6). However, the staining of Foxp3 involves destroying the integrity of cells, which is not suitable for the isolation of live cells. As CD127, a downregulated marker in Tregs, is well correlated to the Foxp3, it is reasonable to speculate that CD127 could be used for the identification of high inhibitive, Foxp3-overexpressing Tregs (7).

Recently, an increasing number of studies indicated that myeloid-derived suppressor cells (MDSCs), a heterogeneous population of myeloid cells, have been featured as a population of cells that involved in the negative regulation of T-cell function (8). According to a literature search, a limited number of studies have been conducted to define the characteristics of MDSCs due to a lack of specific markers. Additionally, limited data are available regarding the different MDSC with suppressor function in patients with cancer, such as head and neck carcinoma, non-small cell lung cancer, colon cancer and 
squamous cell carcinoma $(9,10)$. The phenotype of these cells mainly included $\mathrm{CD} 33^{+}, \mathrm{CD} 34^{+}, \mathrm{CD} 13^{+}, \mathrm{CD} 11 \mathrm{~b}$ and $\mathrm{CD} 15^{-}$. The correlation between MDSCs and Treg in the cancer remains to be elucidated. Previously, human CD14 ${ }^{+}$human leukocyte antigen-antigen D-related (HLA-DR ${ }^{-}$)/low MDSCs induce Foxp $3^{+}$Tregs, whereas no previous studies have been conducted to determine their correlation in breast cancer (11). The aim of the present study was to identify $\mathrm{CD} 4^{+} \mathrm{CD} 25^{+} \mathrm{CD} 127^{-}$Treg cells and $\mathrm{CD} 14^{+} \mathrm{HLA}-\mathrm{DR}-$ /low MDSCs in patients with breast cancer of varying stages, and investigate their roles and the potential interactions in the prognosis of breast cancer.

\section{Materials and methods}

Patients. A total of 40 female patients (mean age 50.7 \pm 4.6 years) with breast cancer were included in the study. A total of 30 healthy individuals (mean age $52.3 \pm 5.8$ years) served as the healthy control. The diagnosis of breast cancer was performed according to the pathological report as revealed in the guidelines proposed by the National Comprehensive Cancer Network (12). Patients with severe organ failure or history of carcinoma were excluded from the study. Individuals on immunosuppressive medication, chemotherapy or undergoing radiation therapy were also excluded. Written informed consent was obtained from each patient. The study protocols were approved by the Ethics Committee of Taicang People's Hospital (Taicang, Jiangsu, China).

Cell isolation. Peripheral blood mononuclear cells (PBMCs) were purified by Ficoll density gradient centrifugation (Biochrom, Ltd., Cambridge, UK) as described previously (13). $\mathrm{CD} 4^{+} \mathrm{CD} 25^{+} \mathrm{CD} 127^{-}$Treg cells were sorted from pre-enriched $\mathrm{CD}^{+}$cells using the BD FACSAria II cell sorting system (BD Biosciences, Franklin Lakes, NJ, USA). CD4 ${ }^{+}$cells were isolated from PBMC using microbeads and the autoMACS separation unit (Miltenyi Biotec, Inc., Cambridge, MA, USA) according to the manufacturer's protocol. The purity of the cells following separation was $>98 \%$.

Flow cytometry. Subsequent to venous blood sample collection, flow cytometry (BD Biosciences) was carried out to determine the marker of the cells. Antibody staining was performed as previously described (14) using an automation workstation (BD Biosciences). The antibodies used were: Fluorescein isothiocyanate (FITC)-CD4 ${ }^{+}$(cat. no. xb01426; Xinbosheng Biotech Co., Ltd., Shenzhen, China), antigen-presenting cells (APC)-CD25+ (cat. no. bcl037; Bichenglan Biotech Co., Ltd., Beijing, China), PE-CD127 (cat. no. 01325; Hengfei Biotech Co., Ltd., Shanghai, China), APC-CD33 ${ }^{+}$(cat. no. 0237; Hengfei Biotech Co., Ltd., Shanghai, China), and PE-CD11b (cat. no. 0542; Yuchang Biotech Co., Ltd., Jinan, China). Following red blood cells lysis, natural Tregs were characterized by the expression of $\mathrm{CD} 4{ }^{+} \mathrm{CD} 25^{+} \mathrm{CD} 127$. Fluorescent-minus-one and isotype controls were used for gating. The purity of cells following sorting was $>98 \%$.

Cytokines plasma concentration. Plasma concentrations of interleukin (IL)-2, tumor necrosis factor- $\alpha$ (TNF- $\alpha$ ), and IL-4 were quantified through fluorescence-activated cell sorting using the CBA kit (BD Biosciences, San Diego, CA, USA)
Table I. Patient characteristics.

\begin{tabular}{lcc}
\hline Variables & Normal control & Patients \\
\hline Total, n & 30 & 40 \\
Age, years & $52.3 \pm 5.8$ & $50.7 \pm 4.6$ \\
Stage I, n & $/$ & 7 \\
Stage II, n & $/$ & 12 \\
Stage III, n & $/$ & 14 \\
Stage IV, n & $/$ & 7 \\
Proximal, n & $/$ & 25 \\
Distal, n & $/$ & 15 \\
Well/moderately differentiated, n & $/$ & 23 \\
Poor differentiated, $\mathrm{n}$ & $/$ & 17
\end{tabular}

Table II. Correlation between Tregs and patient survival.

\begin{tabular}{lcc}
\hline Survival, years & Treg, $\%$ & Treg absolute no., cell $/ \mu 1$ \\
\hline$>5$ & 12.3 & 68.3 \\
$3-5$ & $10.6^{\mathrm{a}}$ & $61.2^{\mathrm{a}}$ \\
$1-3$ & $10.8^{\mathrm{a}}$ & $59.6^{\mathrm{a}}$ \\
$<1$ & $11.2^{\mathrm{a}}$ & $58.7^{\mathrm{a}}$ \\
\hline
\end{tabular}

${ }^{\text {aP }}<0.05$ vs. $>5$-year survival. Treg, regulatory $\mathrm{T}$ cell.

according to the manufacturer's protocol. Intra- and inter-assay coefficients of variation were $<8 \%$.

Statistical analysis. SPSS 17.0 (SPSS, Inc., Chicago, IL, USA) software was used for the data analysis. Measurement data are presented by mean \pm standard deviation, and were analyzed by F-test or Student's t-test. Numerous data were presented as the percentage. $\chi^{2}$ test was performed for the numerous data analysis. $\mathrm{P}<0.05$ was considered to indicate a statistically significant difference.

\section{Results}

Patient characteristics. A total of 40 patients with breast cancer were enrolled in the study, and were categorized into stage I, II, III and IV, respectively. The patients were followed-up until mortality. A total of 30 healthy individuals were enrolled as controls. The patient characteristics are listed in Table I.

Kinetics of Tregs. Natural Tregs were defined as $\mathrm{CD} 4{ }^{+} \mathrm{CD} 25^{+} \mathrm{CD} 127^{-}$cells (Fig. 1 ). The percentage and absolute number of Tregs was higher in breast cancer patients compared to the healthy control. The percentage of Tregs in the patients with stage III or IV breast cancer was higher than those of the stage I or II, respectively (Fig. 2).

For the correlation between Treg kinetics and patient survival, no direct correlation was established between the number or percentage of Tregs and the patient survival (Table II). Of note, the patients with longer life expectancy showed higher Tregs counts and percentages than those without during the follow-up. 

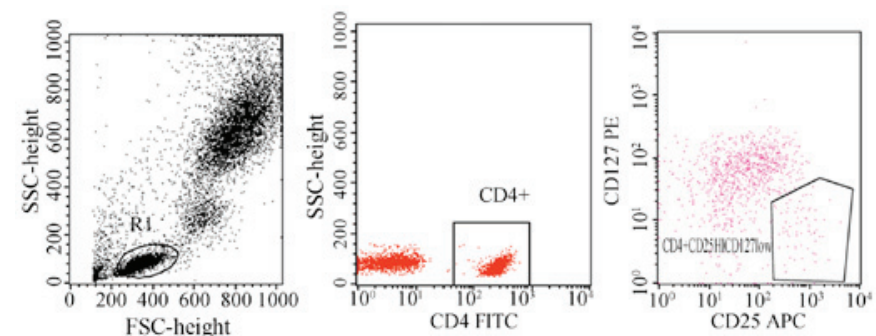

Figure 1. Natural regulatory T cells (Tregs) are defined as $\mathrm{CD} 4^{+} \mathrm{CD} 25^{+} \mathrm{CD} 127$ cells. Flow cytometry was performed for the identification of natural Tregs using the antibodies, including FITC-CD $4^{+}$and APC-CD25 $5^{+}$. All tests were performed at least in triplicate. $\mathrm{CD}$, cluster of differentiation; FITC, fluorescein isothiocyanate; APC, antigen-presenting cells.
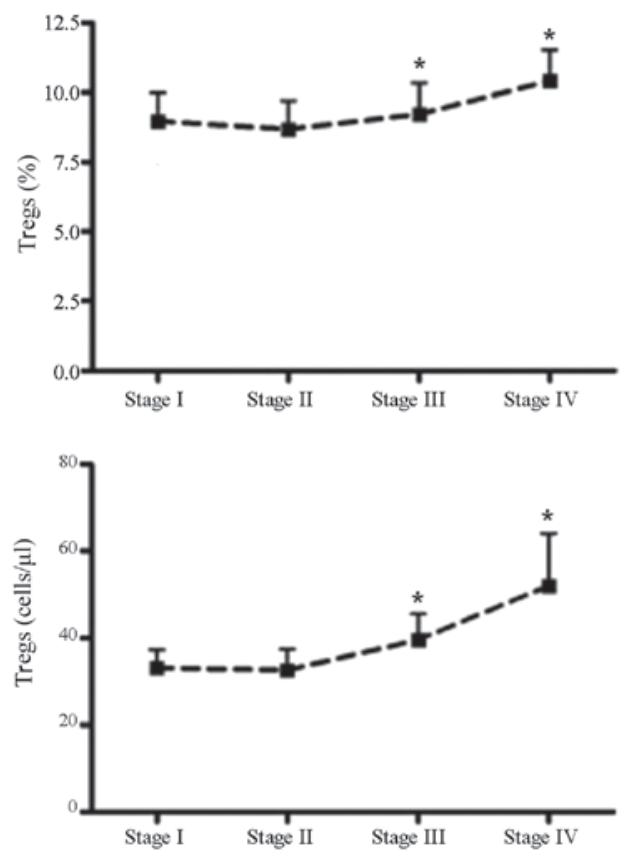

Figure 2. Regulatory T cell (Tregs) kinetics in breast cancer patients. The percentage of Tregs in the patients with stage III or IV breast cancer was higher than those of the stage I or II, respectively. ${ }^{*} \mathrm{P}<0.05$ compared with stage I and II, respectively.
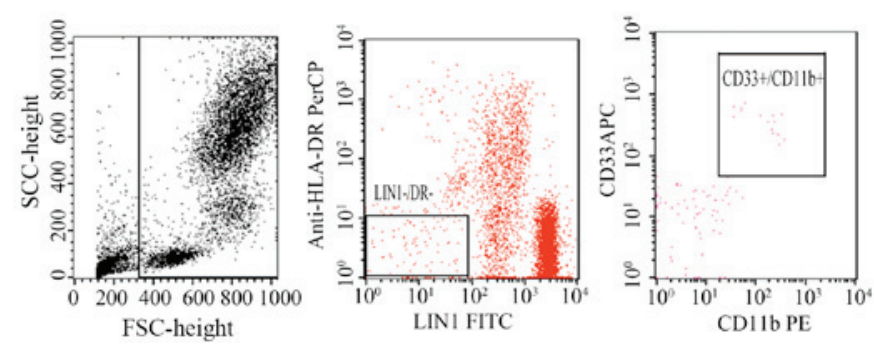

Figure 3. Blood samples obtained from the myeloid-derived suppressor cell frequency are expressed as the percentage of CD33CD11bHLA'DR'LIN ${ }^{-}$. Flow cytometry was performed with the antibodies of PE-Cy5 HLA-DR, PE-CD11b and APC-CD33. All the tests were performed at least in triplicate.

Correlation between Tregs and cytokines. A significant increase was observed in the IL- 2 , TNF- $\alpha$, and IL-4 in the patients compared to the healthy individuals. No statistical difference was observed in the expression of TNF- $\alpha$ in the
Table III. Correlation between Tregs and CD33 ${ }^{+}$MDSCs and CD11b ${ }^{+}$MDSCs.

\begin{tabular}{|c|c|c|c|c|c|}
\hline \multirow[b]{2}{*}{ Tregs } & \multirow[b]{2}{*}{ Total, $\mathrm{n}$} & \multicolumn{2}{|c|}{$\mathrm{CD}^{3} 3^{+} \mathrm{MDSC}$} & \multicolumn{2}{|c|}{$\mathrm{CD}_{11 \mathrm{~b}^{+} \mathrm{MDSC}}$} \\
\hline & & + & - & + & - \\
\hline+++ & 30 & 15 & 10 & 17 & 12 \\
\hline$--/+$ & 10 & 6 & 9 & 5 & 6 \\
\hline Total & 40 & 21 & 19 & 22 & 18 \\
\hline $\mathrm{P}$-value & & \multicolumn{2}{|c|}{$\mathrm{P}<0.05$} & \multicolumn{2}{|c|}{$\mathrm{P}<0.05$} \\
\hline
\end{tabular}

Tregs, regulatory T cells; MDSCs, myeloid-derived suppressor cells.

patients who received treatment compared with those without. No correlation was identified between Tregs and plasma cytokines in the patients and healthy controls.

Kinetics of MDSCs. To determine the percentage and absolute number of MDSCs in patients with breast cancer and healthy individuals, whole blood flow cytometry was performed. Blood samples were obtained from the MDSCs frequency was expressed as the percentage of $\mathrm{CD}_{3} 3^{+} \mathrm{CD} 11 \mathrm{~b}^{+} \mathrm{HLA}$-DR-lineage markers (Lin) ${ }^{-}$. The blood sample obtained from patients was labeled with PE-Cy5 HLA DR, PE-CD11b ${ }^{+}$, and

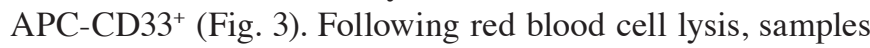
were acquired in a flow cytometer. Subsequently, the obtained cells were gated based on the expression of Lin and MHC class II (HLA-DR). Thus, the MDSCs in this study were defined as $\mathrm{CD}_{33}{ }^{+} \mathrm{CD} 11 \mathrm{~b}^{+} \mathrm{HLR}-\mathrm{DR}-\mathrm{Lin}$. The present results indicated a higher percentage of circulating MDSCs in breast cancer patients compared with the normal individuals (Table III).

Correlation between MDSCs and clinical stage. In the study, the role of MDSCs was also determined in the clinical cancer stage, metastasis and treatment responses. A close correlation was established between clinical cancer stage and percentage and total number of circulating MDSCs. To be exact, a significant increase of MDSC percentage and total number was observed in patients with stage III-IV breast cancer compared with the other cancer patients (stage I-II) and the normal individuals. Patients with stage IV solid tumors showed the maximal mean absolute number and the highest percentage of MDSC, respectively. Compared with the normal individuals, no statistical difference was noticed in the percentage and absolute number of MDSCs in the patients with stage I-II breast cancer regardless of receiving treatment or not (Fig. 4). Furthermore, patients with extensive metastasis tended to have the highest number of circulating MDSCs.

For the patients receiving treatment for breast cancer, higher circulating MDSCs were identified in breast cancer patients receiving ddAC $\left(60 \mathrm{mg} / \mathrm{m}^{2}\right.$ doxorubicin plus $600 \mathrm{mg} / \mathrm{m}^{2}$ cyclophosphamide, $\mathrm{n}=18$ ) chemotherapy compared to those receiving other regimens, including ddT therapy $\left(175 \mathrm{mg} / \mathrm{m}^{2}\right.$ paclitaxel, $\mathrm{n}=22$ ).

Correlation between MDSCs and Tregs in breast cancer patients. The potential correlation between MDSC and Tregs 
A

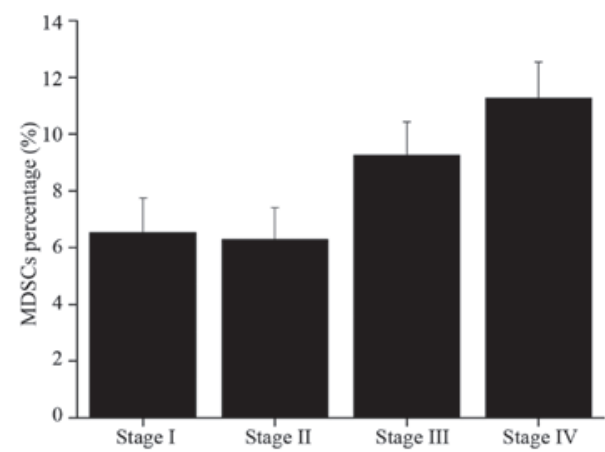

B

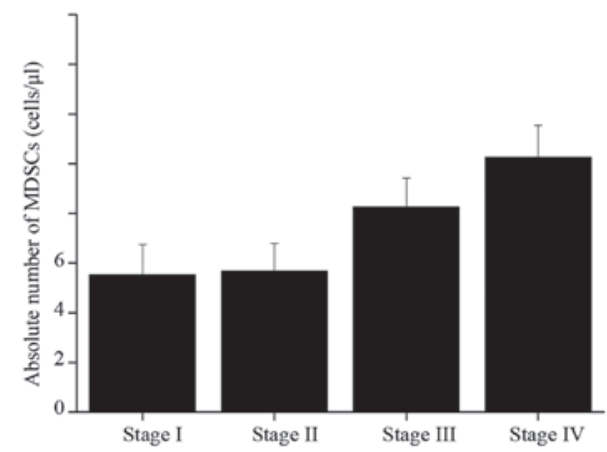

Figure 4. (A) Percentage and (B) absolute number of myeloid-derived suppressor cells (MDSCs) in breast cancer patients of various stages. Patients with stage III and IV breast cancer showed higher percentage of MDSCs compared with those with stage I or II breast cancer.

in the breast cancer patients was also investigated. In the samples with enhanced expression of Tregs, the expression of MDSCs was significantly upregulated in the tumor samples compared with that of the normal individuals.

Correlation between prognosis of breast cancer and Tregs, and MDSCs. No statistical difference was observed in the 3 - and 5-year survival rates in the breast patients with enhanced expression of Tregs, compared with the normal individuals. For the correlation between MDSCs and the patient survival, no statistical difference was noticed in the 3- and 5-year survival rates in the breast patients with enhanced expression of MDSC, compared with the normal individuals. Taken together, Tregs and MDSCs may not be a predictor for the prognosis of overall survival of breast cancer.

\section{Discussion}

Mature myeloid cells are a hallmark of cancer and may have important roles in the tumor evasion originated from immune system. As previously described, the accumulation of MDSCs in animal models and human samples has been reported to be associated with defective dentritic cell function and inhibition of antigen-specific T-cell responses (15). To date, an increasing number of studies have been carried out to determine the phenotype of MDSC in murine models (16), however, limited studies are available to investigate the phenotype of MDSC in humans, particularly patients with breast cancer. According to the previous studies, the Lin HLADR immature myeloid cells in the dendritic cells from cancer patients inhibited the T-cell proliferation and antigen activation, and subsequently, phenotyping of Lin $^{-} \mathrm{HLADR}^{-}$cells were $\mathrm{CD}^{-} 3^{+} \mathrm{CD} 11 \mathrm{~b}^{+}$ cells $(17,18)$. Currently, extensive studies have been performed to investigate the molecules of MDSCs, such as double negative for the MHC class II molecule (HLA DR) and the mature lymphoid or myeloid cells or Lin/Lo $(10,19)$. In the present study, the aim was to investigate the phenotype of MDSCs in patients with breast cancer of various stages. In addition, the correlation between the MDSCs and the prognosis of breast cancer and the clinical stages were determined. Furthermore, the correlation between MDSCs and the expression of Tregs in breast cancer patients was also investigated. A distinct myeloid-suppressor cell population with a phenotype of $\mathrm{CD}^{+} 3^{+} \mathrm{CD} 11 \mathrm{~b}^{+} \mathrm{HLA}^{-} \mathrm{DR}^{-} \mathrm{LIN}^{-}$was isolated from the patients with breast cancer.

The level of MDSC was correlated with clinical stage and metastatic disease burden in patients with breast cancer. For instance, the percentage of MDSCs in whole blood was increased in patients with advanced-stage breast cancer (20). To be exact, the average number of MDSCs in the peripheral blood samples in the patients with stage III or IV was higher than those with stage I or II breast cancer, respectively (21). For the correlation between the MDSCs and the clinical stage, and the prognosis of the breast cancer, the present results revealed that the MDSCs expression was closely associated with the clinical stage of the patients, which was characterized by the increase in the absolute number and the percentage of MDSC in the patients with stage III and IV breast cancer. The present results revealed that MDSC levels in breast cancer patients of all stages were significantly higher compared with the normal controls. Additionally, the percentage and absolute number of increased with the clinical cancer stage and extensive metastasis. Taken together, these results reveal that MDSC is an important mediator of tumor-mediated immune suppression in patients with breast cancers. Furthermore, it may have crucial roles in the immunological tolerance of the breast cancer patients. Although MDSC expression was upregulated in breast cancer patients, no direct correlation was observed between MDSCs and the prognosis of the patients compared to that of the normal individuals.

Tregs have important roles in the control of immune activity against self-antigens. Currently, a large number of Treg subsets have been identified and were reported to inhibit autoimmune and chronic inflammatory responses, such as IL-10-expressing Tregs, and natural $\mathrm{CD} 4{ }^{+} \mathrm{CD} 25^{+}$Tregs (22). To the best of our knowledge, Foxp3 has been identified as a key regulator gene for the development and function of Tregs (23). For example, ectopic expression of Foxp3 in $\mathrm{CD}^{+} \mathrm{CD}^{+} 5^{-} \mathrm{T}$ cells is able to confer suppressive activity in vivo. Foxp3 is known as a reliable marker of Tregs as its expression was not upregulated on T-cell activation. However, the staining of Foxp3 involves destroying the integrity of cells, which is not suitable for the isolation of live cells. In the present study, CD127 was used for the identification of high inhibitive, Foxp3-overexpressing Tregs, and natural Tregs were defined as $\mathrm{CD} 4{ }^{+} \mathrm{CD} 25^{-} \mathrm{CD} 127^{-}$cells. The exact mechanism underlying MDSC-mediated tumor-specific T-cell immune suppression remains to be elucidated. In the present study, MDSCs were significantly upregulated in the tumor samples with enhanced expression of Tregs compared with that of the normal individuals.

As is known to all, Treg cells have an active and significant role in the progression of breast cancer, as well as in the 
suppressing tumor-specific immunity (24). A recent study revealed that the percentages of $\mathrm{CD} 4^{+} \mathrm{CD} 25 \mathrm{~T}$ cells was higher in the peripheral blood mononuclear cells in patients with gastric and esophageal cancer compared to that of the normal individuals (25). Whereas, for the patients who underwent curative resections, the proportions of Treg cells was evidently decreased compared with the baseline levels. In addition, the prevalence of Treg cells in the peripheral blood of gastrointestinal cancer patients was significantly higher than that of the healthy individuals. Furthermore, Liu et al (26) proposed that $\mathrm{CD} 4^{+} \mathrm{CD} 25^{+} \mathrm{CD} 127^{-}$can be used as a selective biomarker to patients with gastric cancer. In addition, among the patients with advanced-stage cancer, those with a higher percentage of Treg cells showed a poor prognosis following chemotherapy. In the present study, the percentage of Treg cells in whole blood was markedly increased in patients with breast cancer. Furthermore, no direct correlation was established between the number or percentage of Tregs and the patient survival. Of note, the patients with longer life expectancy showed higher Treg counts and percentages than those without during the follow-up. For the correlation between MDSCs, Tregs and the patient survival, no statistical difference was identified in the 3- and 5-year survival rates in the breast cancer patients with enhanced expression of MDSC, compared with the normal individuals. On this basis, Tregs and MDSCs may not be predictors for the prognosis of overall survival of breast cancer patients.

In conclusion, patients with breast cancer showed enhanced expression of $\mathrm{CD}^{+} \mathrm{CD} 25^{+} \mathrm{CD} 127^{-}$Tregs cells and $\mathrm{CD}_{3}{ }^{+} \mathrm{CD} 11 \mathrm{~b}^{+} \mathrm{HLA}-\mathrm{DR}$-LIN ${ }^{-}$MDSCs, particularly those with advanced stages cancer. Furthermore, Tregs and MDSCs showed no correlation with the prognosis of breast cancer.

\section{References}

1. Forrest AP, Stewart HJ, Everington D, Prescott RJ, McArdle CS, Harnett AN, Smith DC and George WD; Scottish Cancer Trials Breast Group: Randomised controlled trial of conservation therapy for breast cancer: 6-year analysis of the Scottish trial. Lancet 348: 708-713, 1996.

2. Stewart BW and Kleihues P (eds): World cancer report. In: International Agency for Research on Cancer. IARC Press, Lyon, 2003.

3. Mehta P: Potential role of platelets in the pathogenesis of tumor metastasis. Blood 63: 55-63, 1984.

4. Gilboa E: The risk of autoimmunity associated with tumor immunotherapy. Nat Immunol 2: 789-792, 2001.

5. Mizukami Y, Kono K, Kawaguchi Y, Akaike H, Kamimura K, Sugai $\mathrm{H}$ and Fujii $\mathrm{H}$ : Localisation pattern of Foxp3+ regulatory $\mathrm{T}$ cells is associated with clinical behaviour in gastric cancer. Br J Cancer 98: 148-153, 2008.

6. Allan SE, Crome SQ, Crellin NK, Passerini L, Steiner TS, Bacchetta R, Roncarolo MG and Levings MK: Activation-induced FOXP3 in human T effector cells does not suppress proliferation or cytokine production. Int Immunol 19: 345-354, 2007.

7. Hezova R, Slaby O, Faltejskova P, Mikulkova Z, Buresova I, Raja KR, Hodek J, Ovesna J and Michalek J: MicroRNA-342, microRNA-191 and microRNA-510 are differentially expressed in T regulatory cells of type 1 diabetic patients. Cell Immunol 260: 70-74, 2010.
8. Nagaraj S and Gabrilovich DI: Myeloid-derived suppressor cells. Adv Exp Med Biol 601: 213-223, 2007.

9. Srivastava MK, Bosch JJ, Thompson JA, Ksander BR, Edelman MJ and Ostrand-Rosenberg S: Lung cancer patients CD4(+) T cells are activated in vitro by MHC II cell-based vaccines despite the presence of myeloid-derived suppressor cells. Cancer Immunol Immunother 57: 1493-1504, 2008.

10. Diaz-Montero CM, Salem ML, Nishimura MI, Garrett-Mayer E, Cole DJ and Montero AJ: Increased circulating myeloid-derived suppressor cells correlate with clinical cancer stage, metastatic tumor burden, and doxorubicin-cyclophosphamide chemotherapy. Cancer Immunol Immunother 58: 49-59, 2009.

11. Poschke I, Mougiakakos D, Hansson J, Masucci GV and Kiessling R: Immature immunosuppressive CD14+HLA-DR-/low cells in melanoma patients are Stat $3 \mathrm{hi}$ and overexpress CD80, CD83, and DC-sign. Cancer Res 70: 4335-4345, 2010.

12. Adegboyega TO, Landercasper J, Linebarger JH, et al: Institutional review of compliance with $\mathrm{NCCN}$ guidelines for breast cancer: Lessons learned from real-time multidimensional synoptic reporting. J Natl Compr Canc Netw 13: 177-183, 2015.

13. Ulmer AJ, Scholz W, Ernst M, Brandt E and Flad HD: Isolation and subfractionation of human peripheral blood mononuclear cells (PBMC) by density gradient centrifugation on Percoll. Immunobiology 166: 238-250, 1984.

14. Lyons AB and Parish CR: Determination of lymphocyte division by flow cytometry. J Immunol Methods 171: 131-137, 1994.

15. Nagaraj S and Gabrilovich DI: Myeloid-derived suppressor cells in human cancer. Cancer J 16: 348-353, 2010.

16. Filipazzi P, Huber V and Rivoltini L: Phenotype, function and clinical implications of myeloid-derived suppressor cells in cancer patients. Cancer Immunol Immunother 61: 255-263, 2012.

17. Wang L, Chang EWY, Wong SC, Ong S-M, Chong DQ and Ling KL: Increased myeloid-derived suppressor cells in gastric cancer correlate with cancer stage and plasma S100A8/A9 proinflammatory proteins. J Immunol 190: 794-804, 2013.

18. Mohapatra SS, Bharadwaj SN and Mohapatra S: Compositions and methods for modulating myeloid derived suppressor cells. US Patent 20140336239 A1. Filed December 3, 2011; issued November 13, 2014.

19. Lindau D, Gielen P, Kroesen M, Wesseling P and Adema GJ: The immunosuppressive tumour network: myeloid-derived suppressor cells, regulatory $\mathrm{T}$ cells and natural killer T cells. Immunology 138: 105-115, 2013.

20. Gros A, Turcotte S, Wunderlich JR, Ahmadzadeh M, Dudley ME and Rosenberg SA: Myeloid cells obtained from the blood but not from the tumor can suppress T-cell proliferation in patients with melanoma. Clin Cancer Res 18: 5212-5223, 2012.

21. Lechner MG, Megiel C, Russell SM, Bingham B, Arger N, Woo T and Epstein AL: Functional characterization of human Cd33+ and $\mathrm{Cd} 11 \mathrm{~b}+$ myeloid-derived suppressor cell subsets induced from peripheral blood mononuclear cells co-cultured with a diverse set of human tumor cell lines. J Transl Med 9: 90, 2011.

22. Sakaguchi S: Naturally arising Foxp3-expressing CD25+CD4+ regulatory $\mathrm{T}$ cells in immunological tolerance to self and non-self. Nat Immunol 6: 345-352, 2005.

23. Ramsdell F: Foxp3 and natural regulatory T cells: Key to a cell lineage? Immunity 19: 165-168, 2003.

24. Zhang B, Wang Z, Wu L, Zhang M, Li W, Ding J, Zhu J, Wei H and Zhao K: Circulating and tumor-infiltrating myeloid-derived suppressor cells in patients with colorectal carcinoma. PLoS One 8: e57114, 2013.

25. Hein F, Massin F, Cravoisy-Popovic A, Barraud D, Levy B, Bollaert PE and Gibot S: The relationship between CD4+CD25+CD127- regulatory $\mathrm{T}$ cells and inflammatory response and outcome during shock states. Crit Care 14: R19, 2010.

26. Liu W,Putnam AL,Xu-YuZ,Szot GL,Lee MR,Zhu S, GottliebPA, Kapranov P, Gingeras TR, Fazekas de St Groth B, et al: CD127 expression inversely correlates with FoxP3 and suppressive function of human CD4+ T reg cells. J Exp Med 203: 1701-1711, 2006. 